\title{
Numerical Simulation of Flow over an Airfoil with a Cavity
}

\author{
W. F. J. Olsman* \\ Eindhoven University of Technology, 5600 MB Eindhoven, The Netherlands \\ and \\ T. Colonius \\ California Institute of Technology, Pasadena, California 91125 \\ DOI: $10.2514 / 1 . J 050542$
}

\begin{abstract}
Two-dimensional direct numerical simulation of the flow over a NACA0018 airfoil with a cavity is presented. The low Reynolds number simulations are validated by means of flow visualizations carried out in a water channel. From the simulations, it follows that there are two main regimes of flow inside the cavity. Depending on the angle of attack, the first or the second shear-layer mode (Rossiter tone) is present. The global effect of the cavity on the flow around the airfoil is the generation of vortices that reduce flow separation downstream of the cavity. At high positive angles of attack, the flow separates in front of the cavity, and the separated flow interacts with the cavity, causing the generation of smaller-scale structures and a narrower wake compared with the case when no cavity is present. At certain angles of attack, the numerical results suggest the possibility of a higher lift-to-drag ratio for the airfoil with cavity compared with the airfoil without cavity.
\end{abstract}

\section{Nomenclature}

$c \quad=$ chord of airfoil, $\mathrm{m}$

$D=$ cavity depth, $\mathrm{m}$

$f \quad=$ frequency, $\mathrm{Hz}$

$k=$ reduced frequency

$R e_{c}=$ Reynolds number based on chord length

$S t_{h}=$ Strouhal number based on projected frontal area

$S t_{W}=$ Strouhal number based on cavity opening

$U_{\infty}=$ freestream velocity, $\mathrm{m} / \mathrm{s}$

$W=$ cavity opening, $\mathrm{m}$

$\alpha \quad=$ angle of attack,

$v \quad=$ kinematic viscosity, $\mathrm{m}^{2} / \mathrm{s}$

$\theta=$ boundary-layer momentum thickness, $\mathrm{m}$

$\omega=$ oscillation frequency, $\mathrm{rad} / \mathrm{s}$

\section{Introduction}

$\mathbf{R}$ ECENTLY, the Kasper vortex wing concept has received new attention. The Kasper wing was claimed to achieve higher liftto-drag ratios compared with conventional airfoils (see [1] and references therein). This high ratio was argued to be caused by trapping a vortex (or multiple vortices) in the vicinity of the airfoil at all times. A potential advantage of such a wing is that it can be relatively thick, which is useful from a structural point of view for applications such as high-altitude long endurance (HALE) aircraft or wind turbines. The current work has been performed within a project considering HALE application.

After the original claim of Kasper of high lift-to-drag ratio and trapped vortices, scale models of the Kasper vortex lift wing were tested in a wind tunnel [1]. None of the tested configurations of the vortex wing performed as well as a conventional airfoil. It was suggested that the discrepancy between the wind-tunnel experiments and the claimed flow with trapped vortices was due to the Reynolds number being too low during the wind-tunnel tests.

Received 11 March 2010; revision received 5 August 2010; accepted for publication 7 September 2010. Copyright @ 2010 by the American Institute of Aeronautics and Astronautics, Inc. All rights reserved. Copies of this paper may be made for personal or internal use, on condition that the copier pay the $\$ 10.00$ per-copy fee to the Copyright Clearance Center, Inc., 222 Rosewood Drive, Danvers, MA 01923; include the code 0001-1452/11 and \$10.00 in correspondence with the CCC.

*Ph.D. Student, Department of Physics; w.f.j.olsman@tue.nl.

${ }^{\dagger}$ Professor, Department of Mechanical Engineering. Associate Fellow AIAA.
Although the original claim by Kasper was not supported by the wind-tunnel experiment, theoretical studies have shown that airfoils with trapped vortices can have favorable properties, such as high liftto-drag ratio or prevention of periodic vortex shedding at high angles of attack. It was recently shown that, in a potential flow with two trapped vortices, a nonzero volume body with lift exists with a favorable pressure gradient along the entire contour of the body [2]. A favorable pressure gradient is beneficial, because it prevents flow separation.

Construction of a solution of the flow past an airfoil with a cavity and a trapped vortex is provided by Bunyakin et al. [3,4]. In both papers, the flow is a Batchelor-model flow, which means that the flow is steady, two-dimensional, and the vorticity is uniform inside the region of closed streamlines and zero outside, corresponding to the high Reynolds number limit.

Rectangular cavities in plane walls have been studied extensively [5]. However, not much literature is available for the case of a cavity placed in an airfoil. From the literature about cavities in plane walls, it is known that a cavity can display a shear-layer instability mode that oscillates at a Strouhal number, $S t_{W}=f W / U_{\infty}$, of order unity, where $W$ is the width of the cavity opening, $f$ is the oscillation frequency, and $U_{\infty}$ is the freestream velocity [] $]$. The cavity may also give rise to a cavity wake mode [7], although this mode is rarely observed in planar geometries. In the literature, cavities can be classified as either deep or shallow, depending on the ratio of cavity depth $D$ to cavity opening $W$. Furthermore, one distinguishes for very shallow cavities between open and closed cavities, depending on the reattachment of the flow on the wall within the cavity. For the cavity considered in this paper, $D / W=\mathcal{O}(1)$, which corresponds to an open, shallow cavity.

Because of the approximations made in the theory for designing a wing with a cavity, some of the features of a cavity are omitted, such as the oscillations of the shear layer. The oscillations of the shear layer above the cavity might have a considerable effect on the aerodynamic characteristics of the airfoil in steady as well as unsteady flow. In the case where the opening of the cavity is a significant portion of the chord length of the airfoil, the oscillations of the shear layer might interfere with the shedding of vorticity at the trailing edge. In the current paper, we will focus on the aerodynamics of the airfoil in a steady uniform freestream.

The available theoretical studies mainly focus on approximate theory (inviscid or Bachelor-model flow). In the current paper, we attempt to gain more insight into the flow physics by means of two-dimensional direct numerical simulation (DNS) of the 
incompressible Navier-Stokes equations. Because of the separated nature of the flow and instabilities, Reynolds-averaged NavierStokes computations are not expected to correctly predict the flow. A three-dimensional large eddy simulation (LES) would be expected to capture the flow physics but would be very expensive. Therefore, a preliminary numerical solution would be a two-dimensional DNS. The simulations are qualitatively validated by flow visualizations in a water channel.

In this paper, we will use a geometry that was designed for quick manufacture and low cost. First, the numerical model used will be briefly discussed. Then the results of the numerical simulations for a standard airfoil are presented and compared with experimental data from literature. Hereafter, the computations of the flow around the airfoil with a cavity will be compared with flow visualizations in a water channel and with the numerical results of the standard airfoil.

\section{Numerical Method}

The two-dimensional incompressible Navier-Stokes equations are solved using an immersed boundary (IB) projection method [ㅇ,9]. The solid body of the airfoil is represented, on a regular Cartesian grid, by a set of discrete forces that are in turn regularized (smeared) on the grid. At these discrete body points, the no-slip condition is exactly enforced. The equations are discretized with a second-order finite volume method, and a streamfunction-vorticity approach is used on a staggered grid arrangement. Because of the streamfunction approach, the divergence-free constraint is exactly satisfied (to machine precision). The IB treatment gives rise to a first-order error in the momentum equations near the surface of the body; empirical convergence studies [8] show better than first-order accuracy in the L2 norm. Further details regarding the numerical method can be found in the aforementioned references.

Numerical simulations are performed for several angles of attack $\alpha$, measured in degrees. The angle of attack is defined positive, as indicated in Fig. 1. The standard NACA0018 airfoil is described by 2779 points on its surface, at which the no-slip condition is enforced. For the airfoil with a cavity, this is 2995 points. The properties of the calculations for a positive angle of attack are listed in Table 1. For the airfoil with a cavity, the same cases as listed in Table 1 are also run with the same properties at the corresponding negative angles of attack. The computational domain typically extends to a distance of 12 chord lengths in the upstream and downstream directions and three chord lengths in the upper and lower normal directions. Our IB method uses a series of overlapping consecutively larger and coarser grids; the number of grids is listed in Table 1 as grid levels. The smallest domain with the finest resolution extends to 1.5 chord lengths in the streamwise and 0.35 in the normal directions. The nondimensional grid spacing $\Delta / c$ on this finest grid is $7.4 \cdot 10^{-4}$ for the majority of cases studied. Selected cases are run on a coarser grid with $\Delta / c=1.1 \cdot 10^{-3}$ to test grid convergence. The standard NACA0018 airfoil is computed at four angles of attack: $0,4,10$, and $15^{\circ}$. The computational settings are the same as those listed for the airfoil with a cavity. Typical run times are in the order of $700 \mathrm{~h}$ on a single Advance Micro Devices, Inc. Opteron processor.

In the IB method, the discrete points at which the no-slip condition is enforced cannot be too close to each other. Typically, the distance between those points needs to be equal to or slightly greater than the grid spacing. Note that if the distance between the points is too large, then the surface is porous. At the sharp trailing edge, points of the upper and lower surfaces can be too close to each other. This issue is dealt with by omitting a few points on either the upper or lower surfaces. The standard NACA0018 airfoil without a cavity at a zero

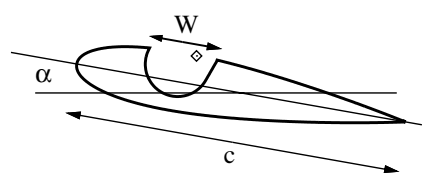

Fig. 1 NACA0018 airfoil with a cavity, with chord length $c=165 \mathrm{~mm}$ and cavity opening of $W=34 \mathrm{~mm}$. A probe location used in the numerical simulations is shown by the tilted square.
Table 1 Setting for NACA0018 cases, with and without cavity-

\begin{tabular}{ccccc}
\hline \hline Angle of attack, & $\begin{array}{c}\text { Smallest } \\
\text { box size }\end{array}$ & $\begin{array}{c}\text { Largest } \\
\text { box size }\end{array}$ & $\begin{array}{c}\text { Grid } \\
\text { levels }\end{array}$ & $\begin{array}{c}\text { Total number } \\
\text { of cells }\end{array}$ \\
\hline 0.0 & $1.49 \times 0.328$ & $23.9 \times 5.25$ & 5 & $4.4 \cdot 10^{6}$ \\
1.0 & $1.49 \times 0.328$ & $23.9 \times 5.25$ & 5 & $4.4 \cdot 10^{6}$ \\
2.0 & $1.49 \times 0.328$ & $23.9 \times 5.25$ & 5 & $4.4 \cdot 10^{6}$ \\
3.0 & $1.49 \times 0.358$ & $23.9 \times 5.73$ & 5 & $4.8 \cdot 10^{6}$ \\
4.0 & $1.49 \times 0.358$ & $23.9 \times 5.73$ & 5 & $4.8 \cdot 10^{6}$ \\
6.0 & $1.49 \times 0.358$ & $23.9 \times 5.73$ & 5 & $4.8 \cdot 10^{6}$ \\
10.0 & $1.49 \times 0.358$ & $23.9 \times 5.73$ & 5 & $4.8 \cdot 10^{6}$ \\
15.0 & $1.49 \times 0.433$ & $47.8 \times 13.9$ & 6 & $6.96 \cdot 10^{6}$ \\
\hline
\end{tabular}

${ }^{\mathrm{a} B o x}$ sizes are indicated in terms of chord lengths.

angle of attack is also computed with a rounded trailing edge where no points are omitted. The trailing-edge radius is $0.3 \%$ of the chord length, which is the same as that of the experimental airfoil. The rounded trailing edge is described by approximately 10 points.

For selected cases, grid resolution and domain size were varied, in order to assess convergence and influence of the far-field boundary. From these results, one can conclude that the results presented, with the resolutions and domain sizes indicated in Table 1, are essentially grid independent. It should be noted that the flows considered show signs of chaotic behavior in vortex shedding. The Reynolds number is sufficiently high such that the formation of large-scale vortices and the subsequent pairing of these structures gives rise to aperiodic lowfrequency oscillations that are difficult to characterize, because the run times are not sufficiently long to observe many periods. Thus, two cases at slightly different resolutions ultimately become decorrelated from each other and contain oscillations over sufficiently long times such that it is not possible to distinguish any possible contamination from the far-field boundaries. However, in all cases, we observed that the time-averaged quantities and qualitative flow regimes are indeed grid independent.

\section{Results}

Two-dimensional simulations are preformed for a standard NACA0018 airfoil and a NACA0018 airfoil with a cavity. The NACA0018 airfoil with a cavity is shown in Fig. 1 . The cavity mouth has $W / c=0.21$. Both edges of the cavity are sharp. The forward sharp edge will fix the separation point, and the rear sharp edge will maximize the feedback loop of the shear layer. This configuration is expected to give the most extreme oscillations of the shear layer.

In all the simulations, the Reynolds number based on the chord length is $R e_{c}=U_{\infty} c / v=2 \cdot 10^{4}$, with $v$ as the kinematic viscosity of the fluid. In the following sections, the results of the computations will be presented and compared with experimental data from literature and flow visualization performed in a water channel. First, the results for the standard NACA0018 airfoil (clean airfoil) are presented, then the results for the NACA0018 airfoil with a cavity are discussed and compared with the standard NACA0018 airfoil and experimental data.

\section{A. NACA0018}

In this section, the results of the numerical simulation of the clean airfoil are presented. At $\alpha=0^{\circ}$, the flow initially separates around $50 \%$ of the chord length. Literature reports separation at $51 \%$ of the chord length from the leading edge for $R e_{c}=1.6 \cdot 10^{5}[10]$. This separation causes a periodic vortex shedding in the wake of the airfoil. We define, here, the Strouhal number $S t_{h}=f h / U_{\infty}$, with $h$ as the projected frontal area of the airfoil. At $\alpha=0^{\circ}, h$ is equal to the thickness of the airfoil and $S t_{h}=0.42$. As the flow develops, the periodic shedding is modulated by a much lower frequency oscillation. The separation points begin to oscillate upstream and downstream, with opposite phases on the upper and lower surfaces. The entire wake is shifted up and down during this low-frequency cycle while its structure is unchanged. Snapshots of the vorticity contours are shown at minimum and maximum lifts in Figs. $2 \mathrm{a}$ and $\underline{2 \mathrm{~b}}$, respectively. For this low-frequency oscillation, $S t_{h} \approx 1.0 \cdot 10^{-2}$ 


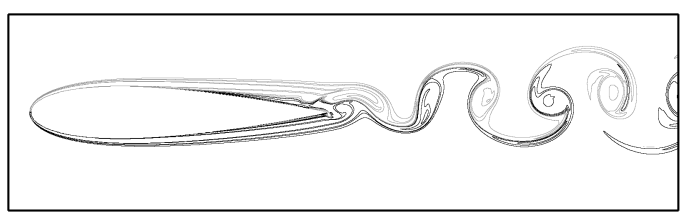

a) At minimum lift force

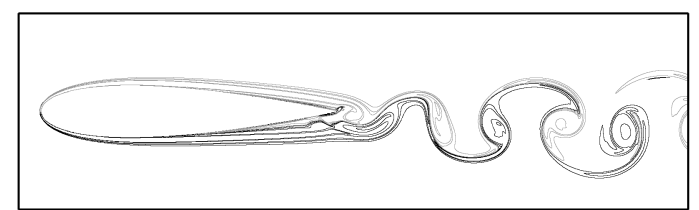

b) At maximum lift force

Fig. 2 Vorticity contour plots for the clean airfoil at $\alpha=0^{\circ}$ at minimum and maximum lifts. Negative vorticity is gray, and positive is black.

and the amplitude of the lift force caused by this low-frequency oscillation is a factor of four larger than the amplitude of the oscillations due to the periodic vortex shedding. Additional calculations have shown that a lower curvature of the trailing edge causes the amplitude of the low-frequency oscillation to decrease by about $10 \%$, but it does not eliminate it. At $\alpha=0.5^{\circ}$, the low-frequency oscillation is also present. A calculation at $R e_{c}=10^{4}$ did not display the low-frequency oscillation. The low-frequency behavior is most likely caused by a unique combination of Reynolds number and geometry. It is likely that this low-frequency behavior is very sensitive to three-dimensional effects and turbulence, which could be a reason why it may not be observed in experiments. There is, however, evidence of similar behavior in literature, but this was reported for airfoils near stall conditions [11].

At $\alpha=4^{\circ}$, the separation point on the suction side moves upstream to about $25 \%$ of the chord length from the leading edge and to $75 \%$ of the chord on the pressure side. Literature on the experimental measurement of the location of the separation point on a NACA0018, at a Reynolds number of $R e_{c}=1.6 \cdot 10^{5}$, shows that at $\alpha=3^{\circ}$, the points of separation on the suction and pressure sides are at 37 and $61 \%$ of the chord length from the leading edge, respectively [10]. The separated boundary layer on the suction side rolls up into large-scale vortices, which are periodically shed downstream. For this periodic vortex shedding, $S t_{h}=0.22$.

At $\alpha=10^{\circ}$ and $\alpha=15^{\circ}$, the flow is similar to the flow at $\alpha=4^{\circ}$, but the separation bubble and the vortex structures are larger, and the separation point on the suction side moves upstream with increasing angle of attack. Also, the separated vortices tend to merge into larger structures before being shed into the wake. At $\alpha=10^{\circ}$, the Strouhal number $S t_{h}$ of the wake is approximately 0.2 .

In Figs. $\underline{3}$ and $\underline{4}$, the time-averaged lift and drag coefficients from the numerical simulation of the clean airfoil are compared with experimental data from the literature [12], at a Reynolds number of $4.14 \cdot 10^{4}$, and more recent experimental data [13], at a Reynolds number of $1.5 \cdot 10^{5}$.

The deviation from the experimental data for the lift coefficient at an $\alpha=15^{\circ}$ is caused by the low Reynolds number and enforced two dimensionality. The separated flow will be three dimensional and turbulent in reality.

The drag coefficient is consistently above the experimental data from the literature; however, the trend is correct. A probable cause for the high values of the drag coefficient is the lower Reynolds number

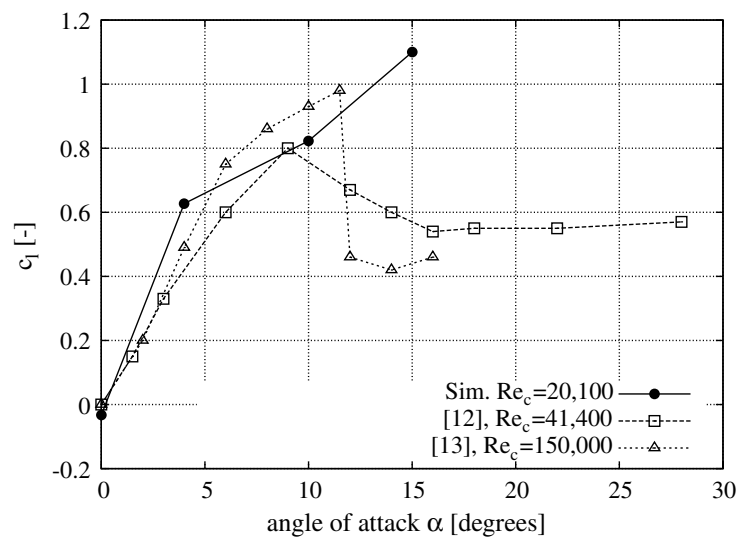

Fig. 3 Time-averaged lift coefficient for the NACA0018 airfoil as a function of the angle of attack. in the numerical simulations. It should also be noted that laminar separation is, in general, very sensitive, even to small disturbances, such as acoustics or freestream turbulence in experimental measurements.

In the IB method, the solution very close to the surface is contaminated by the regularized body forces. Therefore, the pressure in the numerical calculation has been probed at a distance of approximately 1.5 cell spacings from the surface. This will still yield accurate values of the pressure at the surface, since the pressure across the boundary layer is, in the first approximation, uniform.

\section{B. NACA0018 with Cavity}

As already mentioned in Sec. I of this paper, we can expect oscillations of the shear layer above the cavity. To have self-sustained oscillations of this shear layer, the ratio of the momentum thickness of the boundary layer $\theta$ over the width of the cavity opening $W$ should be small [14]. The mainstream velocity used in the calculation of $\theta$ (from numerical data) is the maximum velocity in the boundary layer, which is about $27 \%$ higher than the freestream velocity due to wall curvature. For $\alpha=0^{\circ}, \frac{\theta}{W} \approx 1.6 \cdot 10^{-2}$, which is much smaller than

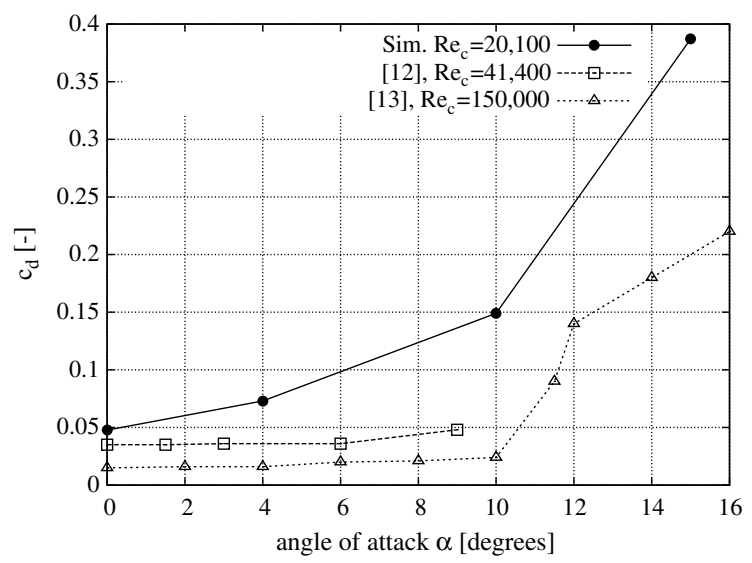

Fig. 4 Time-averaged drag coefficient for the NACA0018 airfoil as a function of the angle of attack.

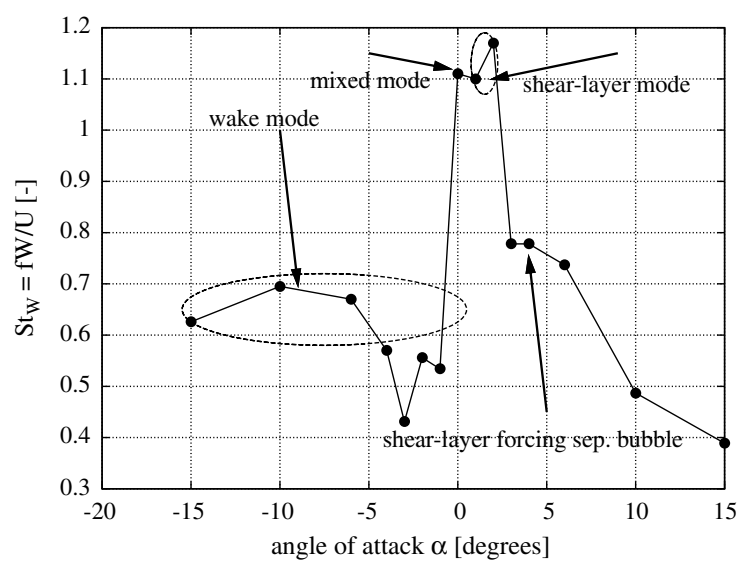

Fig. 5 Values of the Strouhal number of the shear layer $S t_{W}$ above the cavity for the NACA0018 airfoil with a cavity as a function of the angle of attack. 


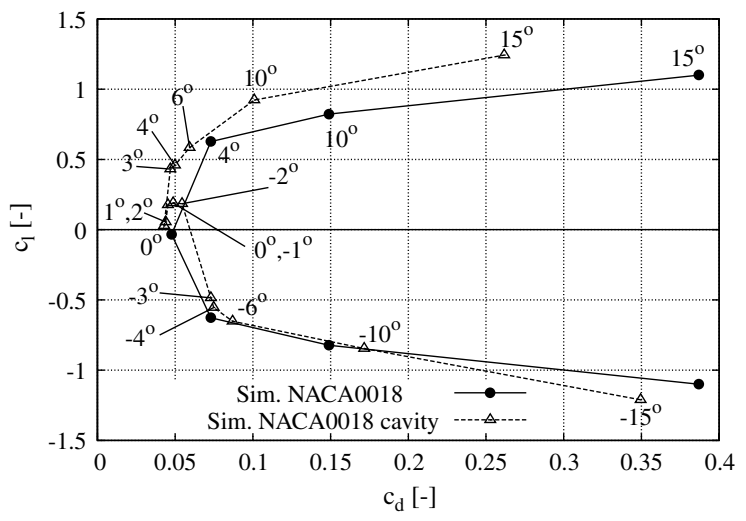

Fig. 6 Lift coefficient as a function of the drag coefficient for the NACA0018 airfoil and the NACA0018 airfoil with cavity. The negative values for the NACA0018 are mirrored points of the positive angles of attack and displayed for easy comparison. The values of the angles of attack are given by the numbers.

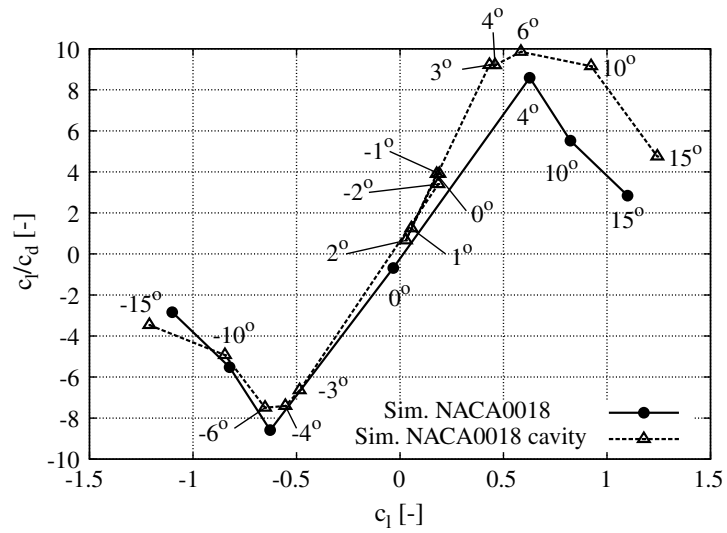

Fig. 7 Lift-to-drag coefficient as a function of the lift coefficient for the NACA0018 airfoil and the NACA0018 airfoil with cavity. The negative values for the NACA0018 are mirrored points of the positive angles of attack and displayed for easy comparison. The values of the angles of attack are given by the numbers.

the required value of about 0.08 , based on linearized stability theory [15].

For the shear-layer oscillations across the cavity, the Strouhal number is defined as $S t_{W}=f W / U_{\infty}$ and is plotted in Fig. 5 for different values of the angle of attack. The probe location is at the rear part of the cavity and is indicated in Fig. 1 with the tilted square. It must be noted that the probe location is the same with respect to the airfoil for all angles of attack. This means that for high positive angles of attack, the probe is not actually in the shear layer but rather inside the separation bubble.

From Fig. $\underline{5}$, it appears that there are two main regimes of the flow inside the cavity. For positive angles of attack, there are two vortices (of opposite sign) inside the cavity, and the shear layer above the cavity weakly interacts with the sharp rear edge of the cavity. In these cases, the Strouhal number based on cavity opening $S t_{W}$ is approximately 1.1 . When compared with literature [6], we conclude that this is the second shear-layer mode.

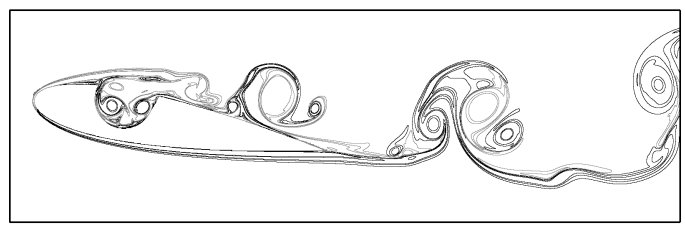

a) Airfoil with cavity
Fig. 8 Vorticity contour plots for the airfoil, with and without a cavity, at $\alpha=10^{\circ}$. Negatity
For $\alpha=3^{\circ}$ and higher, the Strouhal number at the probe location drops down, because the cavity is now fully inside the separation bubble and the shear layer interacts more weakly with the rear sharp edge of the cavity. In this case, however, the separation bubble behavior is forced by the shear layer separating from the upstream edge of the cavity. At $\alpha=10^{\circ}$ and higher, the cavity is fully within the separation bubble. The cavity has a strong influence on the structure of the flow in the separation bubble. It promotes smallerscale vortex shedding than would otherwise occur for the airfoil without a cavity at the same angle of attack.

For negative angles of attack, the shear layer oscillates violently, and vorticity is periodically washed out of the cavity and transported downstream. The Strouhal number of the shear layer at these negative angles of attack is approximately 0.5 , which indicates the first shearlayer mode. For higher negative angles of attack, the Strouhal number increases. This increase can be understood by the flow velocity over the cavity being lower for higher negative angles of attack.

The case of $\alpha=0^{\circ}$ displays a mixed behavior. It starts out as the positive angle of attack with a second shear-layer mode. Two main vortices are present inside the cavity, and the shear layer oscillates weakly. Gradually, the shear layer starts to interact more and more with the sharp rear edge of the cavity and starts to display the more violent first shear-layer mode behavior. After vortex shedding, the flow in the cavity settles down and displays a flow similar to the positive angles of attack again. The flow seems to be switching back and forth between a mild second shear-layer oscillation to a more violent first shear-layer mode oscillation, and back again; this switching back and forth appears to continue. If one applies an oscillating freestream flow, which is oscillating in the direction perpendicular to the airfoil (this generates a velocity field around the airfoil that is equivalent to that of a plunging airfoil), with an amplitude of $5 \%$ of the main flow and a reduced frequency $k=\omega c / 2 U_{\infty}=3.0$, the first shear-layer mode disappears and only the second shear-layer mode is present. For this case, the Strouhal number in the shear layer $S t_{W}$ at the probe location, indicated in Fig. 1, is 1.22 .

In Fig. 6, the lift coefficient, obtained from the simulations, is plotted as a function of the drag coefficient for both the clean airfoil and the airfoil with a cavity. In the upper right part of Fig. $\underline{6}$, where the

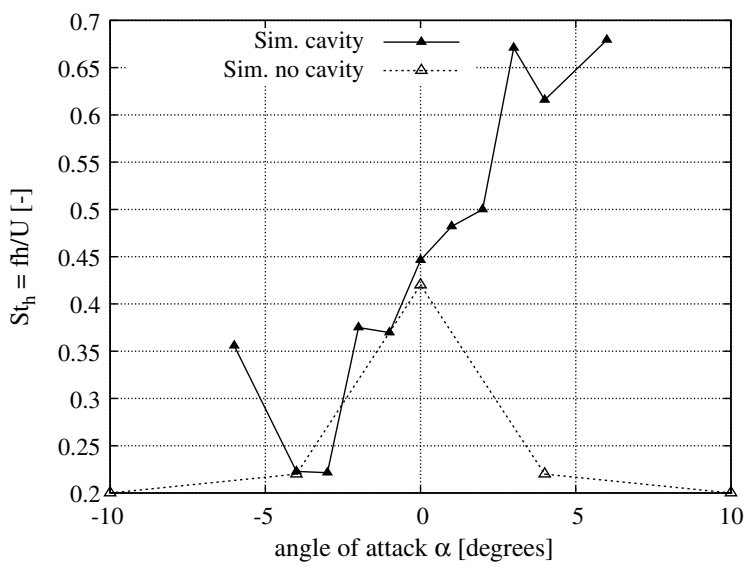

Fig. 9 Strouhal number $S t_{h}$ at a probe location in the wake downstream of the airfoil with a cavity, indicated by the solid line with solid markers, and in the wake downstream of the clean airfoil, indicated by the dotted line with open markers.

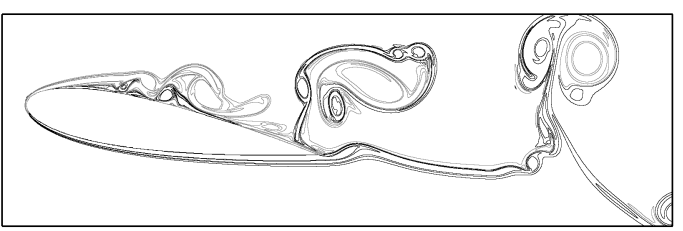

) Airfoil without cavity 


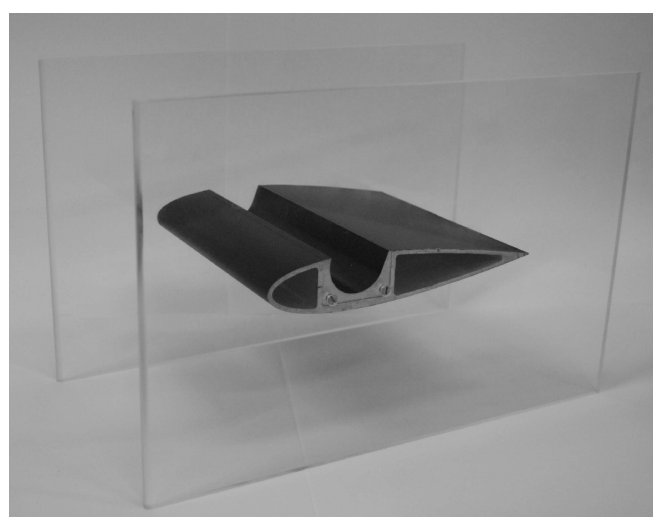

a)

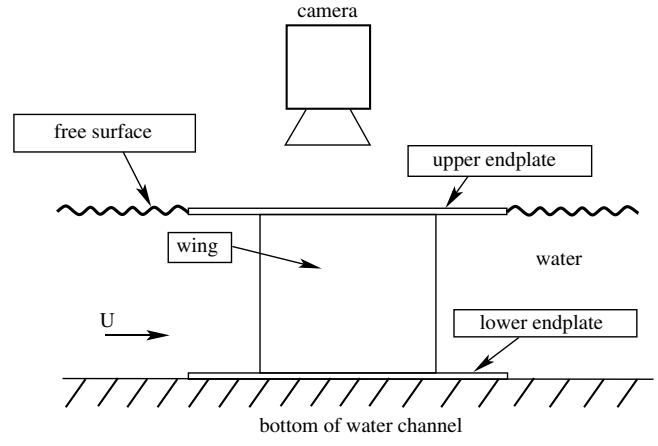

b)

Fig. 10 The airfoil with cavity and endplates is placed vertically inside the water channel: a) photo of the airfoil with endplates and b) a schematic drawing of the setup in the water channel.

lift coefficient is positive, and thus the angle of attack is positive, there are configurations where the lift-to-drag ratio for the airfoil with a cavity is higher than the values for the clean airfoil. From the figure, it appears that the increased efficiency is mainly due to a decrease in drag, since the curve of the airfoil with a cavity is shifted to the left. Figure 7 shows the lift-to-drag ratio as a function of the lift coefficient. This figure shows that, for $c_{l}>0$, the airfoil with a cavity has higher lift-to-drag ratios compared with the clean airfoil. The most significant increase in lift-to-drag ratio is observed for $\alpha=10^{\circ}$. Snapshots of vorticity contours at $\alpha=10^{\circ}$ are shown in Figs. $8 \mathrm{a}$ and $\underline{8 b}$. They show that the wake of the airfoil with a cavity has smaller vortices and is narrower. The vortex dipoles in the wake of the airfoil, in Figs. $8 \mathrm{a}$ and $8 \mathrm{~b}$, are associated with fluctuations in the lift force on the airfoil. Such dipoles have been observed in a previous twodimensional numerical study [16]; however, in experiments at this value of Reynolds number, one may reasonably infer that the wake would become turbulent and modify the coherence and strength of these structures. For example, in the water-tunnel experiments discussed in the next section, these dipoles are not evident in dye visualizations.

Despite these limitations associated with two-dimensional computations, it is interesting to compare the frequency spectra associated with the wake oscillations between the clean airfoil and the airfoil with a cavity. In Fig. 9, the Strouhal number $S t_{h}$ based on frontal projected area $h$ is plotted as a function of the angle of attack at a probe location in the wake of the airfoil with and without cavity, approximately half a chord length downstream of the trailing edge. The frequency was computed from the dominant peak in the power spectrum of the vertical velocity at the probe location.

At positive angles of attack, the airfoil with a cavity shows higher values of $S t_{h}$ compared with the values of the clean airfoil. For example, for $\alpha=4^{\circ}$, the Strouhal number $S t_{h}=0.22$ for the clean airfoil, and $S t_{h}=0.62$ for the airfoil with cavity.

In general, the spectra of the airfoil with a cavity contain more peaks and are broader compared to the cases without a cavity. The careful reader will notice that the results are not plotted for all the values of the angle of attack; this is because it was not possible to distinguish a dominant frequency at these angles of attack. The high Strouhal number at $\alpha=0^{\circ}$ for the clean airfoil is caused by the interaction of the separated boundary layers with each other and the trailing edge. At other angles of attack, the Strouhal number is determined by the separation bubble.

\section{Comparison with Experiments}

For the airfoil with a cavity, no data are available from literature. For a validation of our numerical data, we therefore conducted flow visualizations in a water channel at Eindhoven University of Technology. The water channel used here has a width of $300 \mathrm{~mm}$ and a length of $7 \mathrm{~m}$, in which flows with velocities up to about $25 \mathrm{~cm} / \mathrm{s}$ can be reached. The airfoil section has a spanwise width of $150 \mathrm{~mm}$ and is bounded at the ends by transparent Plexiglas endplates of dimensions $30 \times 20 \mathrm{~cm}^{2}$, and a thickness of $5 \mathrm{~mm}$, to minimize end effects and create quasi-two-dimensional flow over the airfoil; see Fig. 10a. The upstream edges of the endplates are rounded to prevent flow separation, and the airfoil is mounted in the middle of the endplates.

The airfoil is placed vertically in the water channel at a distance of $1.1 \mathrm{~m}$ downstream of the contraction, and the water depth is set to $155 \mathrm{~mm}$. A digital photo camera is mounted above the water surface to capture snapshots of the flow. Figure $10 \mathrm{~b}$ shows a schematic drawing of the setup in the water channel. The flow is illuminated by a horizontal light sheet, which is created by light from two slide projectors that passes through a slit of $3 \mathrm{~mm}$ in black paper. Visualizations are performed by manual injection of fluorescent dye. To observe the shear layer separating the cavity from the main flow, the airfoil is placed vertically in the water channel. The water level is adjusted such that the free surface just touches the upper endplate upon which the airfoil is mounted. This ensures no-slip boundary conditions on both ends of the cavity rather than no slip and free slip when the upper end of the airfoil would stick out above the water surface.

In Figs. 11 and 12, flow visualizations taken at two different instants in time, both at $\alpha=0^{\circ}$ and $R e_{c}=2 \cdot 10^{4}$, are shown on the left and the corresponding vorticity plots of the numerical simulations are on the right. In all the figures, the direction of the flow is from left to right.

The experiments clearly show shear-layer oscillations that are qualitatively similar to those observed in the simulations. $\underline{\underline{\varepsilon}}$ They also show mode switching between the first (Fig. 11) and second (Fig. 12) shear-layer modes at different instants in time. A similar mode switching has been observed for cavities on planar walls [17]. Estimates of the hydrodynamic wavelength $\lambda$ of the structures downstream of the cavity reveal that $\lambda / W \approx 1$ and $\lambda / W \approx 0.5$ for the first and second modes, consistent with Rossiter's [18] observations.

However, the first shear-layer mode appears to be more vigorous in the simulations, with much greater interaction between the shear layer and the vortical structures within the cavity. Without more detailed measurements, it is not possible to draw a firm conclusion, but our expectation is that, consistent with previous two-dimensional simulations of cavity flows [19], the current simulations exaggerate the coherence of the vortical flow in the cavity, whereas the flow in the experiments is likely to be modulated by three-dimensional instabilities of the recirculating flow [20] and by the boundary layers at the spanwise ends of the cavity.

Another difference between the simulations and experiments is observed in the wake of the airfoil, especially at higher angles of attack (not shown). In the experiments, the dye was quickly diffused

${ }^{\S} \mathrm{We}$ are limited to making qualitative comparisons, because dye visualization is not equivalent to vorticity field visualization. Unfortunately, the computational data were not saved at fine enough time intervals to permit detailed particle tracking computations to be made. 


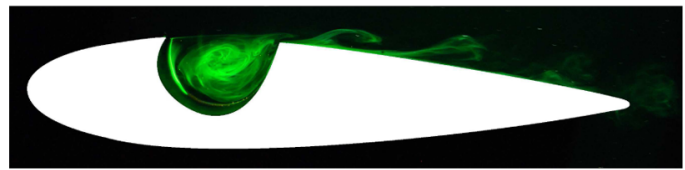

a) Flow visualization

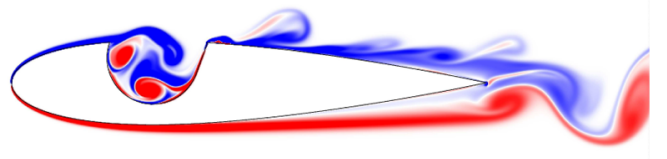

b) Numerical simulation

Fig. 11 Flow visualization of the first shear-layer mode in the water channel and the vorticity contour plot for the airfoil with a cavity at $\alpha=0^{\circ}$ and $R e_{c}=2 \cdot 10^{4}$.

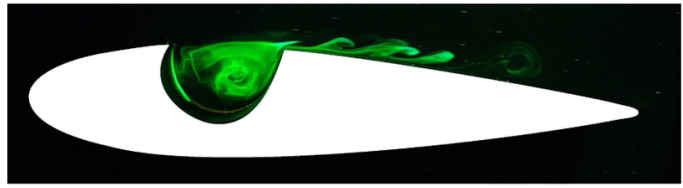

a) Flow visualization

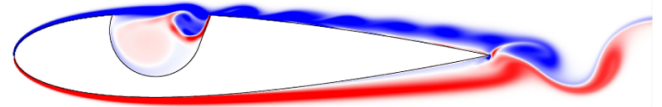

b) Numerical simulation

Fig. 12 Flow visualization of the second shear-layer mode in the water channel and the vorticity contour plot for the airfoil with a cavity at $\alpha=0^{\circ}$ and $R e_{c}=2 \cdot 10^{4}$.

in the wake, presumably due to a transition to turbulent flow that cannot be captured in two-dimensional simulations.

\section{Conclusions}

Two-dimensional DNS results of the flow around a clean airfoil and an airfoil with a cavity have been presented. The main goal of these simulations was to explore the possible flow regimes and to gain more insight into the flow physics. The low Reynolds number simulations of the clean airfoil have been compared with data from the literature and flow visualization carried out in a water channel. In general, the agreement of these simulations with experimental data is reasonable.

The relatively high thickness of the airfoil without a cavity causes a laminar separation, which initially starts approximately half a chord length from the leading edge at $\alpha=0^{\circ}$. Besides the regular vortex shedding due to the separated boundary layers, a very low-frequency oscillation is present at $\alpha=0^{\circ}$. This low-frequency oscillation appears to be caused by a unique combination of geometry and Reynolds number.

For the airfoil with a cavity, the flow in the cavity displays two regimes. For positive angles, the flow in the cavity is dominated by the second shear-layer mode. For negative angles, the flow in the cavity displays behavior that appears similar to a cavity wake mode. For $\alpha=0^{\circ}$, the flow in the cavity switches back and forth between the second shear-layer mode and the wake mode. However, if one applies a small disturbance, the wake mode disappears and only the second shear-layer mode remains. The first and second shear-layer modes were also observed in flow visualizations performed in a water channel.

In general, the oscillations of the shear layer above the cavity generate small vortices, which suppress separation of the boundary layer downstream of the cavity. For $\alpha=0^{\circ}$, the flow on the lower side of the airfoil separates at about $50 \%$ of the chord length from the leading edge, while the presence of the cavity causes the flow to be attached on the upper side; this asymmetry generates a positive lift force.

For $\alpha=4^{\circ}$ and $\alpha=6^{\circ}$, the flow over the airfoil with a cavity separates forward of the cavity. In this case, the shear layer does not impinge on the surface of the airfoil. The shear layer interacts weakly with the sharp rear edge of the cavity, causing a breakup of the shear layer into small-scale structures. In this case, the shear layer is dominating the separation bubble behavior.

At very high angles of attack, the flow over the airfoil with a cavity separates well before the forward edge of the cavity, and the cavity is in the separation bubble. The separated flow displays a strong interaction with the cavity. At $\alpha=10^{\circ}$, this interaction causes the flow to shed smaller-scale structures than the airfoil without a cavity at the same angle of attack. Consequently, the wake is narrower, and the lift-to-drag ratio of the configuration with a cavity is higher compared with the case without a cavity.

The simulations have revealed interesting flow physics associated with the interaction of no less than three different types of instabilities. These are the first- and second-cavity shear-layer modes and separation bubble behavior, which is forced by a shear-layer oscillation. More elaborate experiments and three-dimensional LESs would be a logical next step to obtain more data and physical insight.

\section{Acknowledgments}

The first author would like to acknowledge the European Community for sponsoring this research project under project number AST4-CT-2005-012139. The authors wish to acknowledge G. J. F. van Heijst, A. Hirschberg, R. R. Trieling, and J. F. H. Willems for support, guidance, and constructive suggestions.

\section{References}

[1] Kruppa, E., "A Wind Tunnel Investigation of the Kasper Vortex Concept," AIAA 13th Annual Meeting and Technical Display Incorporating the Forum on the Future of Air Transportation, AIAA Paper 1977-0310, 1977.

[2] Chernyshenko, S., Galletti, B., Iollo, A., and Zannetti, L., "Trapped Vortices and a Favourable Pressure Gradient," Journal of Fluid Mechanics, Vol. 482, 2003, pp. 235-255. doi: $10.1017 / \mathrm{S} 0022112003004026$

[3] Bunyakin, A., Chernyshenko, S., and Stepanov, G., "Inviscid Batchelor-Model Flow past an Aerofoil with a Vortex Trapped in a Cavity," Journal of Fluid Mechanics, Vol. 323, 1996, pp. 367-376. doi:10.1017/S002211209600095X

[4] Bunyakin, A., Chernyshenko, S., and Stepanov, G., "High-ReynoldsNumber Batchelor-Model Asymptotics of a Flow past an Aerofoil with a Vortex Trapped in a Cavity," Journal of Fluid Mechanics, Vol. 358, 1998, pp. 283-297. doi:10.1017/S0022112097008203

[5] Rowley, C., and Williams, D., "Dynamics and Control of HighReynolds-Number Flow over Open Cavities," Annual Review of Fluid Mechanics, Vol. 38, No. 1, 2006, pp. 251-276. doi:10.1146/annurev.fluid.38.050304.092057

[6] Rockwell, D., and Naudasher, E., "Review: Self-Sustained Oscillations of Flow Past Cavities," Journal of Fluids Engineering, Vol. 100, No. 2, 1978, pp. 152-165. doi: $10.1115 / 1.3448624$

[7] Gharib, M., and Roshko, A., "The Effect of Flow Oscillations on Cavity Drag," Journal of Fluid Mechanics, Vol. 177, 1987, pp. 501-530. doi:10.1017/S002211208700106X

[8] Taira, K., and Colonius, T., "The Immersed Boundary Method: A Projection Approach," Journal of Computational Physics, Vol. 225, No. 2, 2007, pp. 2118-2137. doi:10.1016/j.jcp.2007.03.005 
[9] Colonius, T., and Taira, K., "A Fast Immersed Boundary Method Using a Nullspace Approach and Multidomain Far-Field Boundary Conditions," Computer Methods in Applied Mechanics and Engineering, Vol. 197, Nos. 25-28, 2008, pp. 2131-2146. doi:10.1016/j.cma.2007.08.014

[10] Nakano, T., Fujisawa, N., and Lee, S., "Measurement of Tonal-Noise Characteristics and Periodic Flow Structure Around NACA0018 Airfoil," Experiments in Fluids, Vol. 40, No. 3, 2006, pp. 482-490. doi:10.1007/s00348-005-0089-2

[11] Zaman, K., McKinzie, D., and Rumsey, C., "A Natural Low-Frequency Oscillation of the Flow over an Airfoil Near Stall Conditions," Journal of Fluid Mechanics, Vol. 202, 1989, pp. 403-442. doi: $10.1017 / \mathrm{S} 0022112089001230$

[12] Jacobs, E., and Sherman, A., "Airfoil Section Characteristics as Affected by Variations of the Reynolds Number," NACA Rept. 586, 1937.

[13] Timmer, W., "Two-Dimensional Low-Reynolds Number Wind Tunnel Results for Airfoil NACA 0018," Wind Engineering, Vol. 32, No. 6, 2008, pp. 525-537. doi: $10.1260 / 030952408787548848$

[14] Sarohia, V., "Experimental and Analytical Investigation Of Oscillations in Flows over Cavities," Ph.D. Thesis, California Inst. of Technology, Pasadena, CA, 1975.

[15] Michalke, A., "On Spatially Growing Disturbances in an Inviscid Shear
Layer," Journal of Fluid Mechanics, Vol. 23, No. 3, 1965, pp. 521-544. doi: $10.1017 / \mathrm{S} 0022112065001520$

[16] Raju, R., Mittal, R., and Cattafesta, L., "Dynamics of Airfoil Separation Control Using Zero-Net Mass-Flux Forcing," AIAA Journal, Vol. 46, No. 12, 2008, pp. 3103-3115. doi: $10.2514 / 1.37147$

[17] Kegerise, M., Spina, E., Garg, S., and Cattafesta, L., "Mode-Switching and Nonlinear Effects in Compressible Flow Over a Cavity," Physics of Fluids, Vol. 16, No. 3, 2004, pp. 678-687. doi:10.1063/1.1643736

[18] Rossiter, J., "Wind-Tunnel Experiments on the Flow over Rectangular Cavities at Subsonic and Transonic Speeds," Aeronautical Research Council Reports and Memoranda 3438, 1964.

[19] Rowley, C., Colonius, T., and Basu, A., "On Self-Sustained Oscillations in Two-Dimensional Compressible Flow over Rectangular Cavities," Journal of Fluid Mechanics, Vol. 455, 2002, pp. 315-346. doi: $10.1017 /$ S0022112001007534

[20] Bres, G., and Colonius, T., "Three-Dimensional Instabilities in Compressible Flow over Open Cavities," Journal of Fluid Mechanics, Vol. 599, 2008, pp. 309-339. doi: $10.1017 / \mathrm{S} 0022112007009925$

C. Bailly 